\title{
Protocols and Practices in Emergency Evacuation of Women Fleeing Abuse
}

\author{
Patricia Kostouros ${ }^{1}$ and D. Gaye Warthe ${ }^{2}$ \\ 1 Department of Child Studies and Social Work, Mount Royal University, Calgary, Canada \\ 2 Faculty of Health, Community and Education, Mount Royal University, Calgary, Canada
}

Received 02 February 2020

Accepted for publication 19 March 2020

Published 30 June 2020

\begin{abstract}
Over the past several years three major events causing community disasters, two wildfires and a flood, have occurred in the province of Alberta, Canada. When these large-scale events occurred all community residents were asked to follow provincial emergency evacuation procedures, which included a move to an evacuation centre. In some cases, women and their children who were living at domestic violence shelters may have been unsafe since they might encounter the partner they fled at the evacuation site. These researchers encountered such a situation on our campus which is one evacuation centre. In this project, we gathered information from both shelter staff and emergency evacuation personnel about how such circumstances were managed and inquired about future best practices for evacuating women with or without children who are fleeing domestic violence. Interviewees shared insights to inform disaster management and shelter protocols.
\end{abstract}

Keywords: disaster; domestic violence; women's shelter; emergency protocols; emergency evacuation

Between 2011 and 2016 in Alberta, Canada three major events causing community disasters included both wildfires and floods. As reported by the Alberta Emergency Management Agency (AEMA) in the 2011 fire, over 7,000 people were evacuated, over four hundred properties were destroyed leaving over 700 people homeless (AEMA, 2012). In the 2013 floods, several communities were evacuated, including those on First Nations lands. Over 30 communities were impacted, more than 125,000 people were evacuated, including 13,000 people from a municipality where a shelter for women fleeing abuse was flooded (AEMA, 2015). Thirdly, a fire, saw 2,400 homes destroyed with over 88,000 people evacuated (AEMA, 2017). In each of these areas, women's shelters closed, and residents evacuated to centres set up by the AEMA.

When a disaster strikes many people in the community are moved from their present living situations. If a largescale disaster occurs all community residents are asked to follow provincial emergency evacuation procedures, and this often includes a move to and stay at an evacuation centre. Evacuation centres are meant for all community members and therefore, people who use the centres might be known to each other and estranged family members may be confronted when they are relocated to the centre. In some cases, the risk to women who have fled an abusive relationship is higher as they might encounter a partner who is unsafe to them. Thus, fleeing to a safe location is not possible since there are no alternatives.

\section{The intersection of disasters and domestic violence}

There are two concerns that are particularly relevant to the research presented in this paper. The first is the need for domestic violence (DV) shelters and other specialized domestic services to develop protocols, if these do not already exist, that address risks for women and children that would need to access services during and immediately following a disaster. The second is the need to address DV by emergency management systems. At present the majority of current literature focuses on the increased incidence of DV and demands for services following a disaster.

The World Health Organization (2005), identified that violence is an issue that is particularly relevant in communities that have experienced disasters and that more research is needed to fully understand the linkages and potential responses. Factors that are particularly relevant after a disaster mimic DV risk factors noted elsewhere. Specifically, disasters increase vulnerability to violence due 
to increased stress, feelings of powerlessness and loss, the potential for post-traumatic response, the loss of natural support networks and resources that could provide protection, impacts on the economy, and the general lack of resources including resources supporting families impacted by violence (World Health Organization, 2005). The World Health Organization cited several types of DV likely to increase after a disaster including child abuse and neglect and domestic and sexual violence.

In Alberta, the number of women and children who seek shelter in a given year exceeds 10,000 people therefore, it is likely that women and their children will be in a shelter when a disaster strikes. Research (Anastario, Shehab and Lawry, 2009; Enarson and Dhar Chakrabarti, 2009; Harville et al., 2011; Schumacher et al., 2010) showed an increase in the risk of violence for women when a disaster occurs. The challenge of protecting vulnerable women during and after a disaster has surfaced in the literature as early as the late 90s with Enarson's (1999) work with continued investigation presently (First, First and Houston, 2017; Norris, 2014). For example, True (2013) has extended the literature and suggested that gendered violence increases during and after a disaster unless the disaster response community finds ways to address female vulnerability.

There is general consensus about the need for responses that recognize the increased risk of DV following a disaster. Parkinson and Zara (2013) noted that there is significant need for emergency management to attend to DV in the planning, response and recovery stages. However, Parkinson and Zara (2013) suggested that research highlights that DV, from the perspective of emergency management systems, is often "not recognized as a legitimate issue" (p. 32).

The current focus in the literature on the absence of specialized services for DV after a disaster has not addressed that access to DV shelters and to other specialized DV resources can be significantly impacted during the disaster and the evacuation (Gennari et al., 2015). Therefore, the mitigation of risk during and after the disaster needs to be planned for in advance (Enarson, 1999; Gennari et al., 2015). Enarson (1999) and First et al., (2017) suggested that involving DV resources in the preparation for and execution of disaster management would result in increased capacity and improved responses. The present study focuses on the experience of shelter staff relocating women who have fled abuse, during and immediately following disasters resulting in evacuation. Recommendations for improved responses are included.

\section{Policy and procedure limitations}

Disasters affect individuals and communities and when a disaster occurs the impact is not the same for all community members. Physical, socio-economic and gendered differences are some of the variables that will determine who is more vulnerable to impacts of a disaster and recovery
(Fothergill, 1998; 1999; Anastario, Shehab and Lawry, 2009). When a community experiences a disaster, there are usually policies and emergency procedures in place that are expected to be followed by local governments and emergency centres. For example, where people should relocate during the evacuation, and this would be known to all community members, not taking safety of a particular group into consideration. These emergency policies and protocols are mostly universal and are not generally tailored to any one specific population (Laska and Morrow, 2006).

Despite current policies that discuss the needs of the general population, there are limited policies that specifically address the needs of any one specific demographic and in particular the unique needs of women fleeing DV and have been victims of disaster (Laska and Morrow, 2006; Jenkins and Phillips, 2008). This part of the population has no other place to go to avoid the perpetrator who may be in the same emergency evacuation shelter. The local community evacuation centres may be a dangerous place due to inherent privacy and safety concerns. Those who use abuse can locate their partner easily if there is only one designated evacuation centre for an entire community which may have happened in rural areas, such as those in Alberta.

Recent studies (Gilissen et al., 2016) in Europe hypothesized that, despite being limited, a comprehensive evaluative framework that supports objective evaluation would be useful. Kulig et al. (2012) suggested that identifying needs of certain subgroups, such as DV, helps to identify the efficacy of current policies and possible policy changes that might be helpful for victims. Evaluating how well staff are prepared could happen in advance of a disaster, evaluating how well people respond could be done at the time of the disaster or immediately after. Evacuation centre staff could prepare for the unique needs of women fleeing abuse which requires one such evaluative framework. It is important to examine how policies and protocols are being implemented following an actual disaster or emergency situation. Currently, there is little research that looks specifically at this issue despite there being a demand for such an assessment.

\section{Methodology}

One objective of this research was to understand the protocols and practices in emergency evacuation of women fleeing DV, including the potential barriers that may impede a woman's ability to maintain safety. It is important to understand how women's shelters engage in emergency preparedness from a large-scale community perspective, and how they inform emergency evacuation procedures prior to or during a disaster event.

Since we gathered participants in a focus group to talk about their experiences in relation to a particular phenomenon, we conducted a qualitative interpretative study (Merriam, 2002). We were seeking to understand the 
experience of having to follow emergency evacuation protocols for a community disaster, while attempting to maintain safety for women who had already fled abuse. The overarching questions posed in this research were: How aware are the shelter staff of the procedures they are to follow during an emergency evacuation, and how aware are the evacuation centre personnel of the needs of women coming from shelters? In qualitative inquiry, research can follow a particular structure; however, in an interpretive approach the presentation of the content and the structure may vary (Cohen, Kahn and Steeves, 2000). Regardless, there is certain rigour that should be present. According to Fleming, Gaidys and Robb (2002), there are ways in which to establish reliability and thus, we have employed their particular format as a basis for our methodology.

Focus groups are considered appropriate for gathering information related to planning and evaluation (Wilson, 2016). Participants in focus groups can respond to each other's input and take the content deeper and potentially lead to new ideas. Focus groups are deliberate discussions (Rothwell, Anderson and Botkin, 2016) and because of the interaction between participants, knowledge and awareness of the topic increases. Potentially, increased knowledge leads to change in future practice and this focus group process assisted in understanding needed changes to evacuation protocol in the future. This present study was approved by the human ethics research board (HREB) of Mount Royal University in Calgary Alberta, Canada.

\subsection{Present study}

Focus groups were conducted by either one of the authors, or a paid research assistant with training in conducting focus groups. The research assistant was used due to travel convenience. Focus groups were held with shelter staff, one from each area that was impacted by a disaster. Each focus group lasted one to two hours depending on the size of the group. For example, in one area there were three participants and in another area 14 participants. These focus groups were held on the shelter sites in the town or city where the disaster occurred and were all audio recorded. During the focus groups with shelter staff we began by asking about the experience of being evacuated. We then asked about what worked in how the evacuation unfolded, if they knew the procedures they were to follow, and if they practiced emergency evacuation prior to the event and what they would do now, or wish was in place for the future.

We asked similar questions of emergency evacuation centre personnel in terms of knowing if women were arriving from shelters, how their personnel worked to ensure additional safety, if they did, and what they might change to existing protocols to enhance safety if they thought it was relevant. As we did with shelter staff, focus groups were held in the city or town where the disaster occurred. The opportunity presented itself to individually interview a provincial network for emergency social services and therefore, we asked about specific policies for women's shelter evacuation and spoke to one individual. Other questions emerged based on the dialogue produced during the individual interview with this one emergency social services employee.

The interpretive approach to qualitative research typically means that the interviewer will start with an overarching question or two and then questions will emerge based on the content that the participants bring forward (Creswell, 2014). In this case, as noted above, the overarching questions were: How aware are the shelter staff of the procedures they are to follow during an emergency evacuation, and how aware are the evacuation centre personnel of the needs of women coming from shelters? Since additional questions emerge from content, a focus group interview guide was not used.

\subsection{Participants}

This study included groups of staff from shelter programs that had to carry out protocols during the evacuation to an evacuation centre during a disaster. Shelter staff participants were informed of the research opportunity via their connection to the Alberta Council of Women's Shelters (ACSW) and participants from the evacuation centres were recruited via the emergency services programs within the municipalities for the three sites that were affected. In total, there were 19 shelter staff that participated in three focus groups, one per site. A second group of participants were drawn from the evacuation centre personnel. These were people who were in leadership roles or senior management related to emergency evacuation centres, city or town emergency evacuation personnel and a provincial network. In total there were seven evacuation centre personnel involved in interviews and one interview with the provincial emergency social services network, since women fleeing abuse are considered part of the vulnerable sector. No demographic information was collected from any of the participants since we did not believe this information would impact the notion of protocol development.

\subsection{Data analysis}

Data was transcribed using an outside paid resource due to time constraints on the two researchers. Data was managed without the use of software; instead each researcher read and re-read the transcripts for themes that emerged. Researchers acted as inter-raters for each other to confirm themes which were negotiated between the two researchers.

When looking at the transcribed documents it is necessary to draw out the meanings that were presented by the participants. According to van Manen (1990), there are three approaches to uncovering themes: the wholistic approach, the selective approach or the detailed approach. Each approach is unique and will likely yield appropriate results. For this 
research, we combined the above author's suggestions to create a process that resonated with our style more genuinely. For example, we used van Manen's (1990) approach to look at the whole transcript to get a sense of what the participants were describing as their experience. Then we adopted a structure more aligned with the process described by Cohen, Kohn and Steeves (2000) and Creswell (2014), through marking passages and then culling it down to the meanings interpreted from each interview.

\section{Findings}

We describe below the themes that were relevant to all shelters regardless of location or event. In addition to responding to the main research question about evacuating their shelter, staff highlighted some personal issues that had more to do with themselves or their community. We included these themes as it points to the complex factors related to emergency evacuation. In addition, the emergency management personnel provided themes that allow for insights into changes for the future.

\subsection{Time}

One theme that became clear was the notion of time. Several shelter staff knew they had emergency evacuation protocols but also did not have time to concern themselves with whether they were following the protocols precisely. Both shelter staff and emergency evacuation management personnel all agreed that change happened very quickly, and that time was slipping away, "because in the midst of evacuation it's go, go, go, go, go." While time may have been passing quickly it also seemed to stand still when they had to wait for further instruction, "waiting on, you know, the city to let us know where the next rendezvous points are ... because it's not like we can just decide for ourselves." Huang et al. (2017) discussed challenges related to timing during evacuation procedures and recognized that the initiation of evacuation, if timed late, increases risk. Potentially, although the staff all experienced the urgency of the disaster, having to wait for instruction due to the changing circumstances may have increased risk.

\subsection{Communication and safety}

Waiting for instruction and then having instructions change due to changes in the disaster became a challenge for those during the disaster, "and then they kind of flipped and the evacuation centre was evacuated." Several participants, both shelter staff and emergency evacuation personnel, talked about the changing information. For example, in one instance residents were told to go to a muster site, then told to return home only to hear that they did indeed have to leave. As confusion set in and the shelter staff realized they had to leave they were unsure about safety; their own and those of the residents', "where do we go, where is safe?"
Another shelter staff reported that women in the shelter were expected to go to the evacuation centre, yet the shelter remained a safe place to stay. Staff continued to come to the shelter to shower and use laundry facilities as the evacuation facilities were overcrowded and the shelter remained empty. Shelter residents remained in evacuation centres that were not prepared or able to manage their additional safety needs. In an article related to the nursing home population Hyer, Polivka-West and Brown (2007) recognized the challenges related to decisions of staying and sheltering in place or evacuating. One of the challenges discussed is the role of staff in making a decision to leave or stay. When the disaster is looming, safety is the first course of action. However, for some populations sheltering in place may be the more reasonable response, at least initially or until clearer direction is established. If shelter personnel were more involved with disaster management agencies during policy and procedure planning, they may have input into the need for sheltering in place until leaving is necessary.

\subsection{Forfeiting rules}

For the staff attempting to support women fleeing abuse and then having to follow protocols there seemed to be an attitude that when you are in crisis you are going to do what is needed as it unfolds and reaching for a policy manual is unrealistic. To some degree, you might say, "screw policy" as one participant described:

It's just do what you need to do quickly... I think like even with all the protocols that are in place, all the policies, procedures that are in place, when it comes down to the crunch, you jump into survival mode. And you're going to do what it takes to survive. If policy says, no, no, you cannot take the van, or no, no you cannot cross the street, and your only option is to cross the street to get out of there alive, I think you're going to just screw policy.

However, for another participant it was different, she knew that taking the shelter van was against policy since she was not yet at the age where insurance would be valid, seemingly the best solution was not an option. As we listened to this example it occurred to us that even in a disaster sometimes policy is privileged over practicality. Yet we are also keenly aware that had this individual taken the van while not being insured, and had something happened, neither she nor the shelter would have been covered by insurance. This privileged the insurance company policy over the needs of people in the community during an emergency evacuation. Thankfully, someone arrived and could take the residents in the van.

For others, there was no consideration that potentially in a disaster the rules could be forfeited. In the shelter world it is rare that a woman would come into a shelter before she had made contact herself to inquire about an available bed and to have risk assessed. In one case, even during a largescale community evacuation, one shelter expected women 
residents to follow this process. In other words, even though the shelter staff called about beds in another community stating they were being evacuated, the shelter staff in the other community still expected the women to call to be assessed for safety and make their own inquiry about a bed. In a large-scale disaster and evacuation, it may be more helpful that other shelters relax these kinds of rules to make for a smoother transition. However, in one case, due to a personal phone call, the shelter executive director made their own arrangements for transitioning women from one shelter to the other. Had it not been for the personal connection this change in practice may not have been possible.

\subsection{Assumptions}

During the data collection process, we spoke with many people and recognized that there were assumptions that had been made in what people expected. We also heard assumptions about what it means to be in crisis and fleeing abuse. In addition, there were assumptions about who could provide guidance and direction if needed. One of the first assumptions that we heard was that the advocacy organization for women's shelters was a primary point of contact to assist with helping women get to another shelter. While this organization does house a list of shelters in Alberta on their website, it is not their role to act as the conduit for transfer.

Another assumption we heard was that women who were being evacuated from a shelter due to a disaster, would be more concerned about being in a community disaster than the crisis of fleeing abuse. Some people also assumed that the women who were fleeing abuse would not be at heightened risk, that their risk would remain static and not be increased due to the additional crisis. However, the literature refutes this notion (Parkinson and Zara, 2013; Schumacher et al., 2010; World Health Organization, 2016) and recognizes that women are more at risk in a large-scale disaster.

From a disaster management perspective, there was assumptions that women would self-identify if they arrived at an evacuation centre and that shelter staff would identify their needs and it is, "up to the staff or the woman to identify or the agency to say... we have four women who are living in second [stage] housing, you know, when we come, we're going to need space of our own." These personnel also suggested that they would do what they could to provide a separate space if they could. However, because of the volunteer nature and that the evacuation personnel could not identify every situation involving DV, they suggested it was difficult given the nature of the event:

You can come to the evacuation centre it is going to be chaotic...you want to protect that person, because the abuser could also... have been there too, on a day like that, right...I would hate to think that a lady was with a, an abusive situation, and have the husband in the same place, and we wouldn't know.
In one circumstance the evacuation centre contacted a person with DV expertise to manage the evacuation centre. She commented that she was seconded from her usual position, given no direction or instructions and was left to cope as best as she could. In this evacuation centre, the women from the shelter and the staff that had accompanied them were housed in an adjoining complex not visible to the rest of the evacuees. This worked extraordinarily well because of the knowledge the seconded person had about DV.

While there were several assumptions that had been taken up by those experiencing the disasters, often these were embedding in previous experiences. For example, all of the areas that has experienced community disasters have also had several fires or floods that had not ultimately resulted in the need for evacuation.

\subsection{Desensitization}

Each of the communities that were struck by a disaster which resulted in a large-scale evacuation were places where either wildfires or floods were a regular occurrence. As several participants stated, they were used to fires close to their area or water rising that rarely breached the riverbanks. Potentially, residents of those areas had become desensitized that a disaster would occur. It seemed that there was always some threat the never came to fruition and according to these participants, "we never thought it was something that would actually happen," and "people that have been here through many floods...it does sort of desensitizes you to, oh yeah, there's just a flood." When community members have these initial responses, it may decrease the urgency of the situation which could impact the implementation of the existing protocols. If staff in shelters wait until the event has escalated, they may be responding out of panic not process.

\subsection{Personal crisis}

Since most individuals who participated in the study lived in the communities that were evacuated, they had their own personal crisis to manage while also attempting to ensure the safety of the women and children who were in the shelter at the time. These participants were managing the disaster on two levels, personal and professional. On a professional level they followed the procedures as communicated by the emergency evacuation personnel. On a personal level they were wondering about their own family members. In one case it was when the shelter staff received a call from her daughter saying that the fire department was at the house telling them to leave; then she realized how serious the disaster had become. Fortunately, she was able to have her daughter join her, but for others it was less straight forward. For example, in another case the worker was told to go to one place and then, as the fire was coming closer, they were being told to move from there. She was unable to contact her partner to report the change and was preoccupied with where her family was but remained with the shelter residents. Some 
residents dispersed to their own families or returned to partners, but one remained. Eventually, the worker took the resident with her and they went to a friend some distance away where she knew she would be safe, and her family could reunite with her there.

Several stories emerged in our interviews about staff having their own crisis to deal with and shared their own stories of what happened for them once they were relieved from their shelter duties or the women had found alternative places to stay:

A cop tried to stop me, and to, to get us to turn around, because we were on our way to try to get gas, ...I was trying to get to gas and my ex-husband had my kids and he was in front of me. And the cop, right after his SUV went by, the cop tried to say no, turn around, because now there was a fire on that highway. And I said, I'm following my children, and he didn't even try to stop me, he just said, oh you go ahead. Because he knew he was never going to stop me...I'm not going to stop, those are my kids, right.

As we listened to these stories, we were amazed at how dedicated these workers were to the women and children in their care, while concurrently their own homes were being destroyed and their family members were being affected.

4.6.1 Client safety. Although shelter staff were in the midst of their own crisis, they were concerned about resident safety foremost. Shelter staff commented on how difficult it was to know for sure what their clients were going to do once they left them. In one instance a shelter staff took residents to an emergency evacuation centre and made sure they had a separate space and stayed with the women:

We are very concerned about safety and confidentiality for our clients. So, when we got there, we tried to talk with people in charge, right. There were lots of people helping with basic needs, food and blankets, and where to go, but we talked to them and explained that we are vulnerable. We need to have a confidential space, a corner, or a room to be by ourselves.

In this case, the staff member took control and told the emergency evacuation personnel about the required security and they were accommodated. However, she also added that had she not taken charge or disclosed that they were from a women's shelter this may not have occurred. Others commented on the realization that safety now changed for the women who came to them to seek refuge from abuse noting, "once the shelter was closed the protection was gone." Another participant described:

You're coming from a very, very secure facility...You're coming from somewhere that hopefully makes them very safe... it's not just one person that that person is normally fleeing from, it's family members, you know. So anyone, family members in the area who sees that individual [at the evacuation centre] could say to that abuser 'Hey, I saw her here' and an abuser would definitely take that opportunity to, to pounce.

There was concern that the trauma women experienced could be compounded due to multiple safety concerns.

As emergency evacuation personnel reflected on the potential that women who had fled abuse may have been at greater risk, they also provided several suggestions for changes to their system that might increase safety for women who were already fleeing abuse.

\section{Recommendations}

Protocols or guidelines for responding during an emergency is a key characteristic of preparedness. However, having a plan or protocol, as noted by participants, is not in itself adequate to ensure that shelter residents and staff are able to respond in the case of a disaster. The following recommendations are based on the comments of shelter staff, the assessment of emergency personnel, and best practices as identified by the emergency preparedness literature (Enarson, 1997; First, 2014).

\subsection{Planning}

Developing a plan for DV shelters to assist in responding during a disaster should take into consideration the specific context of the disaster, the roles and responsibilities of allied agencies and of organizations responsible for coordinating the emergency response in a region, and the context of the shelter. Much like shelter staff prepare women with a safety plan, First, First and Houston (2017) suggested that both a non-disaster and a disaster safety plan are in order; one which is personalized. For example, as discussed by the participants in this study, finding alternatives for women so that they are less likely to think they need to return to a partner, and be with them again, during the disaster.

\subsection{Responses}

The type of disaster or emergency may dictate the type of response required. Although shelter staff were not able to comment on whether a different response was required whether the disaster was a flood or fire, as none of the staff interviewed had experienced both, anticipating how the shelter would need to respond differently for specific types of disasters is consistent with emergency preparedness plan recommendations. For example, participants shared their frustration with attempts to locate alternate shelter beds for residents when floods were threatening as staff were relying on practices typically used when the shelter was at capacity. This typically included contacting shelters in close proximity to determine availability of space. During a disaster when all residents need to be evacuated, this approach to re-housing is not practical nor efficient particularly when all shelters in an area are impacted. 
In addition, other specialized resources may be unavailable to women evacuated from shelters. Specifically, specialized cultural resources or counsellors, such as Elders, may be absent due to their own evacuation. Although accessible, some responses may not be culturally appropriate or sensitive. As noted by one of our Indigenous participants, "they tried to bring in some Elders to deal with some of the issues they were facing, but it was quite difficult for the Elders. A lot of the Elders were flooded as well." The responses typically relied on were unavailable and this was not a consideration in emergency preparedness.

\subsection{Coordination and communication}

The need for a group or organization that could assist with coordination and communication between shelters needing to be evacuated and other shelters outside the region not impacted by the emergency was identified in the research. That no organization currently has such a role during disasters does not preclude shelters from identifying an agency with expertise in DV that could take on coordination, communication and advocacy during a disaster. While this recommendation for the ACWS to take a pivotal role is Alberta specific, this is easily generalized to other communities that have a collaborative shelter network.

\subsection{Protocol development}

When developing a disaster protocol specific to residents in women's shelters those included should be disaster responders and providers, including the evacuation centres along with shelter networks. Working together to anticipate challenges and develop a plan for the evacuation of vulnerable populations, such as residents of DV shelters, does not eliminate risk but can contribute to increased knowledge of the complexities of an evacuation and how best to manage the risks for the complex needs of shelter residents. First, First and Houston (2017, p. 394) suggested that "developing connection before a disaster is important because it provides time to create trust and understanding between organizations ahead of an emergency." Reviewing existing protocols in evacuation centres in collaboration with shelter networks can help to identify specific aspects of the protocol requiring modifications for risk populations. Planning for the needs of women and families experiencing DV increases the knowledge capacity of disaster responders and providers (First, 2014).

A protocol also needs to reflect the unique context of the shelter and the community. For example, a shelter in a small community may require a protocol recognizing the potential for heightened risk in an evacuation centre because community members are known to each other. All shelters need independent protocols that address a process for making decisions, a communication strategy, a plan for identifying vulnerable populations within the shelter, reflect cultural considerations, present an alternate evacuation plan for shelter residents, and address implementation and both initial and ongoing training needs. Potentially, a shelter disaster protocol may differ from the direction posed by a regional emergency management group and they should be supported in the flexibility to respond differently if it means increased safety. Therefore, there may be a need for changes to policy not just protocols or procedures.

\subsection{Policy change}

When proposing policy change it is helpful and realistic to see it more as policy enhancement (Henstra, 2010; 2011). At present, emergency management recognizes vulnerable populations and note that some are disproportionately impacted in a disaster. However, this does not include DV in particular. If current policies are not taking the needs of women leaving abusive situations into consideration, then it may be necessary to enhance existing policies. Emergency management policy is a unique domain that encompasses a course of action that public authorities adhere to during times of disasters and states of emergency. It is an organized set of steps that are to be taken to deal with a disaster situation to minimize risk, injury and financial damage. Emergency policies normally change or evolve when the policy no longer sufficiently meets the needs for which it was designed. When policies are lagging, then governments and communities once informed, can make changes to existing policies. Research (Ollerenshaw, Graymore and Mcdonald, 2016) showed that local governments play a significant role in supporting their communities when a disaster strikes.

Although Canada has numerous federal emergency and disaster agencies each with a unique role in disaster preparedness, Sadiq, Tharp and Graham (2016) suggested that people tend to rely more on local agencies versus federal agencies when disasters strikes. This information is useful because it allows future research to target the agencies that will have the most impact when a disaster occurs and to ensure preparedness at all levels of government and community. Supporting federal emergency management is beneficial however, being able to focus on local agencies that provide direct services is perhaps even more critical, particularly after the disaster.

\subsection{Post-disaster recovery and advocacy}

Post-disaster recovery provides an opportunity to visit the process of responding to an emergency evacuation. It is not unusual for systems to gather reports on the effectiveness of responses and lessons learned. These often include recommendations for future planning. We suggest that these reports include feedback from vulnerable sectors, including the women's shelters.

As mentioned by some of the participants in this study, the initial and temporary disaster supports start to disappear, and long-terms needs remain high. For women fleeing abuse 
housing is already a concern and when there is a shortage of homes in a community following a disaster, this makes resourcing more difficult. In addition, funds for families after a disaster can make it difficult for women to secure funds if the policy limits financial support to one household. Advocacy for the specific needs of women and their children, given their vulnerability after a disaster is vital.

\section{Limitations}

Some general challenges that we faced in completing this research was in relation to the length of time that had expired since the disaster event and the number of participants in one of the focus groups. For example, one of the disasters took place in 2011 and we conducted this research in 2017. Therefore, many of the staff had already left the town and those now in staff positions at the local women's shelter were not present at the time of the evacuation. However, we were able to locate some staff who were able to meet for a focus group, but their recall was limited. Having only three participants in this focus group may not have provided as much insight into the recommendations. For example, we may have found other themes or additional insight into the themes that were found.

The disaster that occurred in 2016 happened only one year prior to this research and staff were still attempting to manage their own personal suffering and therefore, has some difficulty being able to think critically about the event and the protocols. For the disaster in 2013, it was four years after the event, many staff members were available, and they were able to think critically about the event and had created sufficient emotional distance.

A final limitation was that we did not collect demographics. While initially we did not think demographics could lead to meaning making about protocols in this area, it did occur to us after collecting data, that some demographic information might have made a difference in how participants took up the challenges related to the disasters. For example, when participants talked about having been desensitized to floods and fires, due to the frequency of these in their communities, length of time living in those communities or where in the community they lived when previous floods or fires occurred may or may not have played a part in their desensitization. In addition, age may have made a difference, with those who were older having had more experiences working in crisis. Those experiences may or may not have made a difference in managing both the crisis for the women in their care as well as for themselves and their own families.

\section{Conclusions}

It is clear that women fleeing DV may need additional supports when in a community disaster and need to evacuate from a women's shelter. Effective policy that weaves the needs of women fleeing abuse into the structures of emergency management are essential. The vulnerability of women when a disaster occurs has been shown to increase, and policy related to the vulnerable sector needs consideration in disaster planning and post-disaster concerns. Those who work with women fleeing abuse and understand their specific needs could advocate on their behalf recognizing the added stressor of having made an attempt to leave and abusive relationship, only to have to flee the safety of the shelter.

A final comment in addition to assisting women who flee abuse is consideration of safety for other marginalized or vulnerable groups. For example, many populations are at risk of DV or intimate partner violence such as trans or nongender binary people, men or Elders. In addition, other populations that may not experience violence could also be more vulnerable in an emergency evacuation situation such as those with English as an additional language or other barriers such as cognitive delay. While it may be difficult to account for every population that needs services in an emergency evacuation, heightening our awareness in relation to DV may also lead to protocol development for the more vulnerable other.

\section{References}

Alberta Emergency Management Agency (2012) Lesser Slave Lake regional urban interface: lessons learned, Final report. Available

http://www.aema.alberta.ca/documents/0426-LessonsLearned-Final-Report.pdf (Accessed: 8 April 2018)

Alberta Emergency Management Agency (2015) Review and analysis of the government of Alberta's response to and recovery from 2013 floods. Available at: http://www.aema.alberta.ca/documents/2013-flood-responsereport.pdf (Accessed: 8 April 2018)

Alberta Emergency Management Agency (2017) May 2016 Wood Buffalo wildfire: post-incident assessment report. Available at: https://www.alberta.ca/assets/documents/Wildfire-KPMGReport.pdf (Accessed: 15 April 2018)

Anastario, M., Shehab, N. and Lawry, L. (2009) 'Increased gender-based violence among women internally displaced in Mississippi 2 years post-Hurricane Katrina', Disaster Medicine and Public Health Preparedness, 3(1), pp. 18-26.

Cohen, M., Kahn, D. and Steeves, R. (2000) Hermeneutic phenomenological research: A practical guide for nurse researchers. Thousand Oaks, CA: Sage.

Creswell, J. (2014) Research design: Qualitative, quantitative and mixed method approaches. Thousand Oaks, CA: Sage.

Enarson, E. (1997) Responding to domestic violence and disaster: Guidelines for women's services and disaster practitioners. Vancouver: University of British Columbia. Disaster Preparedness Resource Centre. Available at: 
http://gdnonline.org/resources/dv-and-disaster.doc (Accessed: 15 April 2018)

Enarson, E. (1999) 'Violence against women in disasters: A study of domestic violence programs in the United States and Canada', Violence Against Women, 5(7), pp. 742-768.

Enarson, E. and Dhar Chakrabarti, P. G. (ed.) (2009) Women, gender and disaster: global issues and initiatives. Thousand Oaks, CA: Sage. doi.org/10.4135/9788132108078

First, J. (2014) Disaster and domestic violence: A factsheet for disaster responders and providers. Disaster and Community Crisis Center, University of Missouri. Available at:

https://dcc.missouri.edu/assets/doc/dcc_domestic_violence_a nd_disasters.pdf (Accessed: 6 June 2018)

First, J. First, N. and Houston, J. (2017) 'Intimate partner violence and disasters: A framework for empowering women experiencing violence in disaster setting', Affilia: Journal of Women and Social Work, 32(3), pp. 390-403.

Fleming, V., Gaidys, U. and Robb, Y. (2002) 'Hermeneutic research in nursing: Developing a Gadamerian-based research method', Nursing Inquiry, 10, pp. 113-120.

Fothergill, A. (1998) 'The neglect of gender in disaster work: an overview of the literature' in Enarson, E. and B. H. Morrow (eds.) The gendered terrain of disaster: Through women's eyes. Westport: Praeger, pp. 11-25.

Fothergill, A. (1999) 'An exploratory study of woman battering in the Grand Forks flood disaster: Implications for community responses and policies', International Journal of Mass Emergencies and Disasters, 17(1), pp. 79-98.

Gennari, F., Arango, D., Urban, A. and McCleary-Sills, J. (2015) Violence against women and girls: disaster risk management brief. Global Women's Institute: George Washington University. Available at: https://www.alnap.org/help-library/violence-against-womenand-girls-disaster-risk-management-brief (Accessed: 8 May 2018)

Gilissen, H. K., Alexander, M., Matczak, P., Pettersson, M. and Bruzzone, S. (2016) 'A framework for evaluating the effectiveness of flood emergency management systems in Europe', Ecology and Society, 21(4), pp. 27-42.

Harville, E. W., Taylor, C. A., Tesfai, H., Xiong, X. and Buekens, P. (2011) 'Experience of Hurricane Katrina and intimate partner violence', Journal of Interpersonal Violence, 26(4), pp. 833-845.

Henstra, D. (2010) 'Evaluating local government emergency management programs: what framework should public managers adopt?', Public Administration Review, 70(2), pp. 236-246.

Henstra, D. (2011) 'The dynamics of policy change: A longitudinal analysis of emergency management in Ontario, 1950-2010', Journal of Policy History, 23(3), pp. 399-428.
Huang, S-K., Wu, H-C., Lindell, M., Wei, H-L. and Samuelson, C.D. (2017) 'Perceptions, behavioral expectations, and implementation timing for response actions in a hurricane emergency', Natural Hazards, 88(1), pp. 533558.

Hyer, K., Polivka-West, L. M. and Brown, L. (2007) 'Nursing homes and assisted living facilities: Planning and decision making for sheltering in place or evacuation', Generations, 4, pp. 29-33.

Jenkins, P. and Phillips, B. (2008) 'Battered women, catastrophe, and the context of safety after Hurricane Katrina', NWSA Journal, 20(3), pp. 49-68.

Kulig, J., Pujadas Botey, A., Townshend, I., Awosoga, O., Shepard, B., Edge, D. and Smolenski, S. (2012) Report of the School Survey: Slave Lake, AB. Lethbridge: University of Lethbridge. Available at: https://hdl.handle.net/10133/3269 (Accessed: 8 May 2018)

Laska, S. and Morrow, B. H. (2006) 'Social vulnerabilities and Hurricane Katrina: An undisaster in New Orleans', Marine Technology Society Journal, 40(4), pp. 16-26.

Merriam, S. (2002) Qualitative Research in Practice. San Francisco, CA: John Wiley and Sons.

Norris, F. H. (2014) Disaster and domestic violence. National Center for PTSD. Available at: http//www.ptsd.va.gov/professional/trauma/disasterterrorism/disaster-domestic-violence.asp (Accessed: 22 Apr. 2018)

Ollerenshaw, A., Graymore, M. and Mcdonald, K. (2016) 'Beyond the call of duty: The integral role of rural local government in emergency management', Rural Society, 25(3), pp. 185-203.

Parkinson, D. and Zara, C. (2013) 'The hidden disaster: Domestic violence in the aftermath of disaster', Australian Journal of Emergency Management, 28(2), pp. 28-35.

Rothwell, E., Anderson, R. and Botkin, J. (2016) 'Deliberative discussions focus groups', Qualitative Health Research, 26(6), pp. 734-740.

Sadiq, A., Tharp, K. and Graham, J. D. (2016) 'FEMA versus local governments: influence and reliance in disaster preparedness', Natural Hazards, 82(1), pp. 123-138.

Schumacher, J. A., Coffey, S. F., Norris, F. H., Tracy, M., Clements, K. and Galea, S. (2010) 'Intimate partner violence and Hurricane Katrina: Predictors and associated mental health outcomes', Violence and Victims, 25(5), pp. 588-603.

True, J. (2013) 'Gendered violence in disasters: Learning from New Orleans, Haiti and Christchurch', Aotearoa New Zealand Social Work, 25(2), pp. 78-89.

van Manen, M. (1990) Researching lived experience: Human science for an action sensitive pedagogy. Albany, New York: State University of New York Press. 
Wilson, V. (2016) 'Research methods: Interviews', Evidence Based Library and Information Practice, 11(1), pp. 47-49.

World Health Organization (2005) Violence and disasters. Department of Injuries and Violence Prevention: World Health Organization, Geneva. Available at: https://www.who.int/violence_injury_prevention/publication s/violence/violence_disasters.pdf (Accessed: 5 June 2018)

World Health Organization (2016) Violence against women: Intimate partner and sexual violence against women. Available at: http://www.who.int/mediacentre/factsheets/fs239/en/ (Accessed: 5 June 2018) 